\title{
Ion distributions in the vicinity of Mars: Signatures of heating and acceleration processes
}

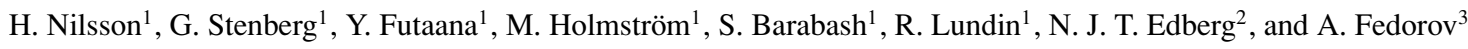 \\ ${ }^{1}$ Swedish Institute of Space Physics, Kiruna, Sweden \\ ${ }^{2}$ Swedish Institute of Space Physics, Uppsala, Sweden \\ ${ }^{3}$ Centre d'Etude Spatiale des Rayonnements, Toulouse, France
}

(Received February 9, 2011; Revised April 28, 2011; Accepted April 28, 2011; Online published March 8, 2012)

\begin{abstract}
More than three years of data from the ASPERA-3 instrument on-board Mars Express has been used to compile average distribution functions of ions in and around the Mars induced magnetosphere. We present samples of average distribution functions, as well as average flux patterns based on the average distribution functions, all suitable for detailed comparison with models of the near-Mars space environment. The average heavy ion distributions close to the planet form thermal populations with a temperature of 3 to $10 \mathrm{eV}$. The distribution functions in the tail consist of two populations, one cold which is an extension of the low altitude population, and one accelerated population of ionospheric origin ions. All significant fluxes of heavy ions in the tail are tailward. The heavy ions in the magnetosheath form a plume with the flow aligned with the bow shock, and a more radial flow direction than the solar wind origin flow. Summarizing the escape processes, ionospheric ions are heated close to the planet, presumably through wave-particle interaction. These heated populations are accelerated in the tailward direction in a restricted region. Another significant escape path is through the magnetosheath. A part of the ionospheric population is likely accelerated in the radial direction, out into the magnetosheath, although pick up of an oxygen exosphere may also be a viable source for this escape. Increased energy input from the solar wind during CIR events appear to mainly increase the number flux of escaping particles, the average energy of the escaping particles is not strongly affected. Heavy ions on the dayside may precipitate and cause sputtering of the atmosphere, though fluxes are likely lower than $0.4 \times 10^{23} \mathrm{~s}^{-1}$.
\end{abstract}

Key words: Mars, solar wind interaction, ion escape.

\section{Introduction}

Mars is the smallest of the three terrestrial planets with atmospheres, with the weakest gravity and the thinnest atmosphere. The difference in solar wind interaction between Earth and Mars (as well as Venus) is primarily the presence of a strong internal magnetic field at Earth, which can balance the pressure of the solar wind at a distance of about $10 R_{\mathrm{E}}$. Mars does not have a significant internal magnetic field, but some significant crustal magnetic fields which does play a local role in the solar wind-atmosphere interaction (Acuña et al., 1998; Connerney et al., 2001; Brain et al., 2005; Edberg et al., 2008).

At the unmagnetized planets a pressure balance is formed between the dynamic pressure of the solar wind and the ionosphere supported by induced magnetic fields caused by ionospheric currents. The pressure balance occurs through the intermediary of the piled-up magnetic field in the magnetosheath, see for example Luhmann (1990) for a tutorial. For Mars the ionosphere balances the external pressure at an altitude of a few $100 \mathrm{~km}(200-800 \mathrm{~km}$ with median $380 \mathrm{~km}$ for Mars, Mitchell et al. (2000)). The atmospheres of Earth and Mars are also very different, but this appears to play

Copyright (c) The Society of Geomagnetism and Earth, Planetary and Space Sciences (SGEPSS); The Seismological Society of Japan; The Volcanological Society of Japan; The Geodetic Society of Japan; The Japanese Society for Planetary Sciences; TERRAPUB.

doi:10.5047/eps.2011.04.011 less role from a plasma dynamics point of view. In a denser atmosphere the ionosphere forms at a higher altitude, in a less dense atmosphere at lower altitude, but the area of the ionosphere as seen from above is not significantly affected, as the altitude of the ionosphere is small compared to the planetary radius. Atmospheric and exospheric scale height, as well as exobase and homopause levels will influence both details of the interaction and possibly the relative loss of different atmospheric species. For details on solar wind influence on the evolution of atmospheres we refer to Lammer et al. (2008) and references therein.

There is some evidence that Mars was once rather similar to Earth, with oceans and a denser atmosphere. Much of the evidence comes from geological features indicating outbursts of liquid water, as well as in situ samples of sedimentary rocks (Squyres et al., 2004). Some studies have questioned the picture of a wet and warm early Mars, e.g. Hoffman (2000). Measurements of planetary origin ion escape from Mars due to solar wind interaction has been made during high solar activity with the ASPERA instrument onboard the Phobos-2 spacecraft. These measurements indicated that a substantial amount of the ancient Mars atmosphere and oceans, if the denser atmosphere and oceans ever existed, could have been removed by solar wind interaction (Lundin et al., 1989; Verigin et al., 1991). Low solar activity loss rates based on ASPERA-3 measurements are of the order of $10^{24} \mathrm{~s}^{-1}$ (Lundin et al., 2008b; Nilsson et al., 
2011), an order of magnitude less than the Phobos-2 high solar activity results, and thus not a significant contribution to the total escape.

In order to generalize the results obtained at present day Mars, so that they can be applied to the early solar system and exoplanets, we must investigate the details of the ion escape processes, not just present escape rates. The higher escape rates measured with Phobos- 2 indicates a substantial dependence on the solar cycle. Such a dependence can come from enhanced production of ions and increased atmospheric/ionospheric scale height due to increased solar EUV, i.e. a change of the ionospheric source, as well as from more energy available for removal of atmospheric particles. The response to changing solar wind and EUV conditions during the declining phase of a solar maximum and solar minimum have been studied by Lundin et al. (2008a) and Nilsson et al. (2010). The response of the ion escape and the shape of the induced magnetosphere to changing solar wind flux and solar EUV could be qualitatively described for current conditions. Edberg et al. (2010) and Nilsson et al. (2011) looked at times when co-rotating interaction regions (CIR) arrived at Mars, and found that the outflow increased by a factor of on average 2.5 during a CIR, significantly less than the enhancement for solar maximum implied by the Phobos 2 measurements. The influence of magnetic anomalies on ion distributions have also been studied, e.g. Lundin et al. (2011) and Nilsson et al. (2006a, 2011), finding that the magnetic anomalies does affect the ion fluxes in the vicinity of the planet, but does not cause a hemispheric asymmetry of the escape inside the nominal IMB. The escape from the dayside ionosphere and/or pick up ion fluxes are larger from the northern hemisphere, which have less magnetic anomalies. In this study we will present average distribution functions of ions, obtained using almost 4 years of data, to study in detail the ion distribution, flow and acceleration in the vicinity of Mars. These results can be compared with model results, to elucidate topics such as the source of the escaping ions (ionosphere or exosphere), the location and nature of acceleration regions, and the response of the escape to increased energy input into the atmosphere system of Mars.

\section{Instrument and Data}

We use data from the Ion Mass Analyzer (IMA) of the ASPERA-3 instrument package on Mars Express (Barabash and the ASPERA-3 team, 2006). Mars Express is placed in an elliptical orbit with a periapsis altitude of about $275 \mathrm{~km}$, an apoapsis altitude of about $10000 \mathrm{~km}$ and a period of $6.75 \mathrm{~h}$. IMA has an energy coverage of $10 \mathrm{eV}$ to $36 \mathrm{keV}$. The lower energy limit of the measured source population is affected by spacecraft velocity (ram-effect) and spacecraft potential. The instrument has an energy resolution of $7 \%$ and steps through 96 energy levels in $12 \mathrm{~s}$. Before May 2007 the instrument did in practice not measure below $30 \mathrm{eV}$. Between May 2007 and mid November 2009 an energy table going down to $10 \mathrm{eV}$ was used. After that an improved table going down as close as possible to zero is in use. We have used data from May 2007 until February 2011.

The intrinsic field-of-view of IMA is $4.5^{\circ} \times 360^{\circ}$ in the spacecraft $X-Z$ plane, divided into 16 azimuthal sectors of $22.5^{\circ}$ (see Barabash and the ASPERA-3 team, 2006) their figure 37 or Nilsson et al. (2006a) their figure 1 for a schematic description of the IMA field-of-view as well as a definition of the spacecraft coordinate system). Mars Express is a three-axis stabilized instrument platform. In order to obtain a limited three-dimensional field-of-view IMA utilizes an electrostatic entrance deflection system to bring particles in from about $\pm 45^{\circ}$ from the viewing plane of the instrument. The entrance deflection is stepped through 16 different elevation angles with an angular spacing close to $5.625^{\circ}$ to obtain a total angular coverage of $90^{\circ}$ out of the instrument viewing plane. Part of the three-dimensional field-of-view is blocked by the spacecraft body and the solar panels. Throughout our work we use only data from viewing directions which are not affected by spacecraft blocking. We use a conservative approximation of the blocking due to the moveable solar panels, rather removing too much than too little data. At energies below $50 \mathrm{eV}$ the entrance deflection system has insufficient resolution to obtain the desired angles of deflection, and therefore the entrance deflection system is set to a value as close to zero deflection as possible. This means that below $50 \mathrm{eV}$ the measurements are two-dimensional.

The mass per charge of observed ions is determined through a magnetic deflection system and a micro-channel plate based position detection system. Ions are distributed over 32 mass anodes, and a given ion mass will give counts spread around a given mass anode for a given energy. Through fitting of theoretical response curves for the major heavy planetary origin ions, assumed to be $\mathrm{O}^{+}, \mathrm{O}_{2}^{+}$and $\mathrm{CO}_{2}^{+}$, these can be separately determined. The separation is better at low energy, below a few $100 \mathrm{eV}$, where the relative trajectory difference in the magnetic field at a given energy is larger, facilitating mass separation. Nominal positions for protons and alpha particles are always well separated from the heavier ions. The mass resolution of IMA is discussed in more detail in Carlsson et al. (2006).

The data used in this study has been treated by a noise removal process which removes counts with all zero neighbors in the nearest mass end energy channels. Elevated background counts, caused by solar EUV which may enter for certain conditions, or high energy particles, are automatically removed. Proton fluxes may for certain conditions, especially for intense fluxes at energies below $1 \mathrm{keV}$, contaminate mass channels corresponding to other ion species. This gives rise to a characteristic signal which is detected and accounted for in the data used in this study. Such data is not just removed, it is used to estimate the proton flux. The automatic algorithm detects more than $90 \%$ of the spread out protons, and essentially all cases with fairly strong proton fluxes. For weaker proton fluxes the spread out proton signal is not as wide as usual, but concentrated in a spot in the middle of the detector range. This coincides fairly well with where the $\mathrm{O}^{2+}$ mass peak is located for such energies. We calculate the flux in the mass channels corresponding to $\mathrm{O}^{2+}$, and increased fluxes in this mass range in the magnetosheath at energies typical for proton contamination can be used as a signature of insufficient removal of proton contamination. If the heavy ion flux is not much 
larger than the flux at the $\mathrm{O}^{2+}$ mass peak we discard the heavy ion data, in order to ascertain that we have a local maximum at the heavy ion mass channels, and thus remove the remaining contamination from protons. Such data is not used to reconstruct the proton fluxes. Sample energy spectra with background noise and proton contamination were shown and discussed in Fränz et al. (2006).

A final aspect of our data treatment is cross-talk between different view directions (sectors) of the instrument. This appears to occur only for rather strong fluxes in some sectors, in which case a weak signal may be detected in all azimuthal sectors at the same energy as the original signal. In average distribution functions obtained over many years a rather clear but quite weak ring is seen at the same energy as the strongest signal. Real ring distributions, due to classical pick-up of ions in the solar wind, would not occur at the same energy for all viewing directions in the spacecraft reference frame. We have in our data subtracted the 25th percentile of the flux in the different angular directions for each energy level above $100 \mathrm{eV}$. This lessens the visual impact of the rings in the plots of the average distribution functions, but does not otherwise have a significant impact on any of our obtained results. Below $100 \mathrm{eV}$ this type of rings are not discernible, and the measured fluxes have a wider angular spread so that this method is not necessary.

\section{Data Analysis Method}

The IMA angular coverage is not only limited, it is potentially strongly biased as the orientation of the three-axis stabilized platform is determined by the needs of the on-board cameras and other instruments designed to study the surface and atmosphere of Mars. The orientation is therefore neither evenly nor randomly distributed. The directional sampling problem is further emphasized by the low energy ion data, below $50 \mathrm{eV}$, which does not have entrance deflection scanning and is thus two-dimensional. Things are further complicated by the fact that the space environment around Mars is not symmetric, it shows clear asymmetry relative to the direction of the solar wind electric field (Dubinin et al., 2006; Fedorov et al., 2006; Barabash et al., 2007; Nilsson et al., 2010). The crustal fields have a very uneven distribution between the hemispheres (Acuña et al., 1998; Connerney et al., 2001), which may also bias data if these are important for the ion distributions around Mars and the ion outflow.

We have chosen to deal with this problem by binning the IMA data into angular bins as well as spatial bins. Our spatial binning is based on the Mars Solar Orbit coordinate system, with $X_{\mathrm{MSO}}$ pointing from the center of Mars towards the sun. $Y_{\text {MSO }}$ is the component of the sun velocity relative to Mars orthogonal to $X_{\mathrm{MSO}}$, i.e. in the orbit plane, pointed towards dusk. $Z_{\mathrm{MSO}}$ completes a right handed system, with $+Z_{\text {MSO }}$ towards north. This basic coordinate system has been transformed to a two dimensional cylindrical coordinate system with the coordinates $X_{\mathrm{MSO}}$ and $R_{\mathrm{MSO}}\left(=\sqrt{Y_{\mathrm{MSO}}^{2}+Z_{\mathrm{MSO}}^{2}}\right)$.

For each spatial position we have binned the data after the angle of arrival of measured ions. The angle of arrival is measured relative to the $+X_{\text {MSO }}$ direction. The three- dimensional solid angle contributing to each angular bin is the solid angle of a cone-section with the angular width of the two-dimensional angular bin. The angle relative to $X_{\mathrm{MSO}}$, i.e. the sun direction, is given a sign such that it is positive along the $R_{\mathrm{MSO}}$ vector, i.e. outward from the cylinder center. The solid angle for each angular bin thus corresponds to half a solid angle cone, one in for each hemisphere in the $R$ direction. The solid angle contributing to each angular bin is used in the calculation of the total three-dimensional flux.

We therefore get average distribution functions for different spatial positions around the planet. Each angular direction bin and spatial bin have a different amount of samples. The average is calculated separately for each direction, thereby taking into account uneven angular sampling. Finally we calculate the energy shift due to the spacecraft motion for each individual looking direction of the instrument. This will at times move the measurable ions down to lower energies, more so for heavier ions. The ram velocity shifted data is interpolated to an energy table which consists of the energy table used between May 2007 and mid-November 2009, which goes down to $10 \mathrm{eV}$ energy, with 10 equally spaced extra energy levels $1 \mathrm{eV}$ apart below $10 \mathrm{eV}$. We do not take the spacecraft electric charge into account. The spacecraft charge is likely to be positive for a sunlit spacecraft in a tenuous plasma and likely negative in a dense plasma. For directional fluxes with a drift energy well above the energy corresponding to a spacecraft charge the flux estimates will not be affected, the ions will be slowed down/accelerated but the flux will remain constant. Similarly, cold plasma accelerated into the instrument by a negative charge will to a first order approximation not affect the net directional flux measured by the spacecraft, as the extra contribution will be the same from all directions. The spacecraft charge can sometimes be estimated using observations of photoelectron peaks of known energy, e.g. Fränz et al. (2006). For ionospheric measurements the spacecraft potential is typically in the range -10 to $0 \mathrm{~V}$.

The average distribution functions have been calculated for three heavy ion species, $\mathrm{O}^{+}, \mathrm{O}_{2}^{+}$and $\mathrm{CO}_{2}^{+}$. The mass separation works well for low energy ions, which is where the separation is most needed, in order to compensate for the spacecraft motion. Apart from that effect, the estimate of the number flux is not dependent on the assumed ion mass. For high energy the mass separation is less reliable, so we present all data as the sum of the flux of all heavy ions. The average distribution functions at low energy, i.e. mostly close to the planet, can be well fitted by a drifting Maxwellian. We have done so for the low energy data, thereby extrapolating to lower energies than those observed.

In the final analysis presented in this paper we used spatial bins of size $0.2 \times 0.2 R_{\mathrm{M}}$ in our $X_{\mathrm{MSO}}-R_{\mathrm{MSO}}$ coordinate system. The angular bin size was $20^{\circ}$, approximately matching the resolution of the instrument.

3.1 Fitting of distribution functions to low energy data

Inspection of the average distribution functions at low energy (below about $100 \mathrm{eV}$ ) shows a close to Maxwellian distribution, an example is shown in Fig. 1. The sample shown is for $\mathrm{O}^{+}$, the distribution functions of the other heavy ion species have a similar shape. For the heavy ion species we 

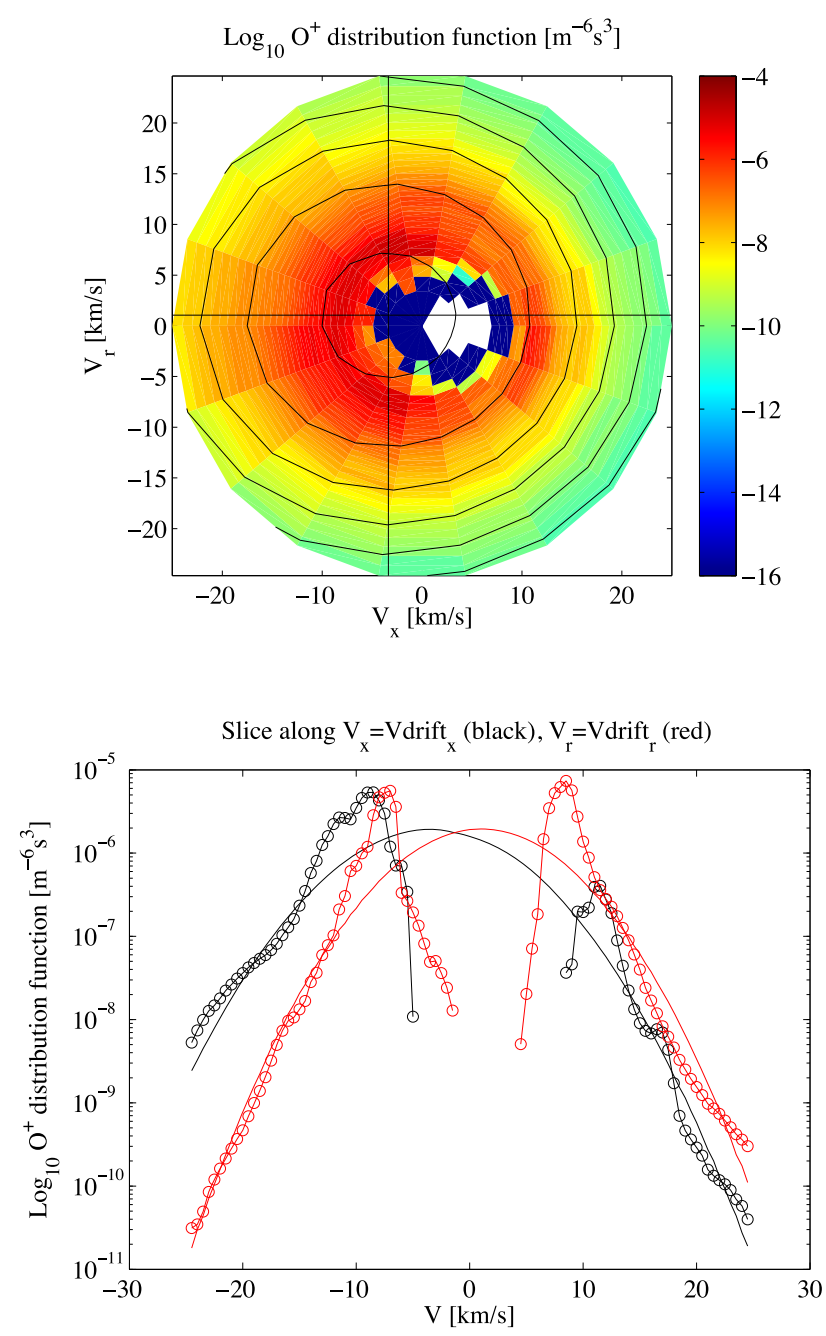

Fig. 1. Statistical distribution of $\mathrm{O}^{+}$from the sample region 6 as shown in Fig. 2. The upper panel shows the logarithm of the average distribution function $\left(\mathrm{m}^{-6} \mathrm{~s}^{3}\right)$ as a colour scale, with a fitted two-dimensional Maxwellian shown as black contour lines. Straight vertical and horizontal black lines indicate the centre of the fitted distribution. Slices of the distribution function along the vertical and horizontal lines are shown in the lower panel, with black for the vertical line $\left(V_{x}=V_{\text {drift } x}\right)$ and red for the horizontal line $\left(V_{r}=V_{\text {drift } r}\right)$. Solid lines show the fitted function, while circles connected with a solid line show measured data.

have therefore fitted drifting two-dimensional Maxwellian distributions to the data below $50 \mathrm{eV}$, i.e. for the energy range where the IMA data is two-dimensional. A least square fit of five unknowns (coefficients for $V_{x}, V_{r}, V_{x}^{2}, V_{r}^{2}$ and a constant) to the logarithm of the average distribution function values allows for the reconstruction of drift velocity components $V_{\mathrm{drift} x}$ and $V_{\mathrm{drift} r}$, the thermal velocities in $X_{\mathrm{MSO}}$ and $R_{\mathrm{MSO}}$ directions and the density of a Maxwellian distribution. The sample average distribution function was measured in the terminator region, just inside the nominal IMB and is marked as sample region 6 of Fig. 2. The upper panel of Fig. 1 shows the logarithm of the average distribution function $\left(\mathrm{m}^{-6} \mathrm{~s}^{3}\right)$ as a colour scale, with the fitted function as black contour lines. Distribution functions obtained from IMA and fitting of moment functions to the data is discussed in Fränz et al. (2006). Straight vertical and horizontal black lines indicate the centre of the fitted distribution. Slices of the distribution function along the
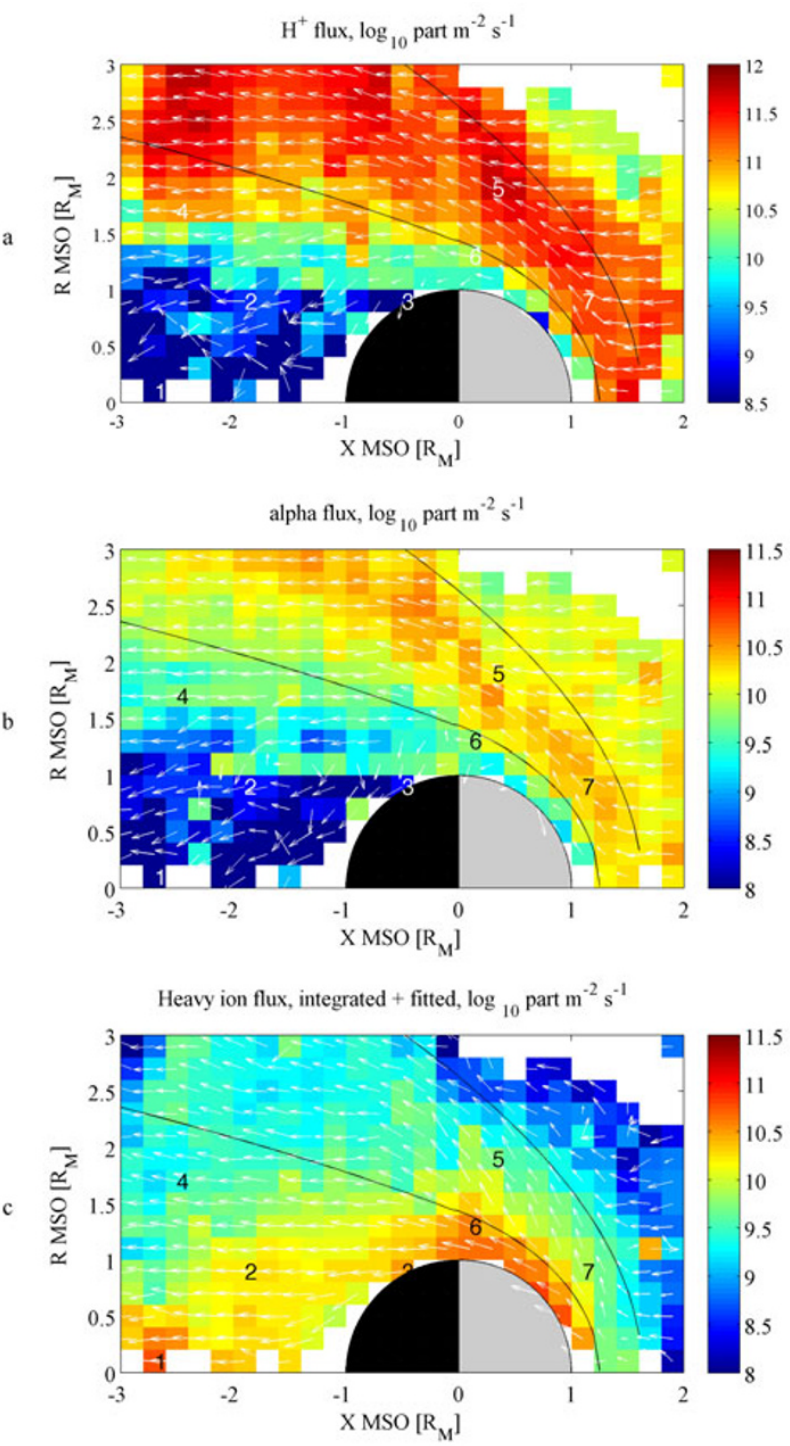

Fig. 2. Flux of ions in the near-Mars space in $X_{\mathrm{MSO}}$, $R_{\text {MSO }}\left(\sqrt{Y_{\text {MSO }}^{2}+Z_{\text {MSO }}^{2}}\right)$ coordinates, the colour scale indicates particle flux density $\left[\mathrm{m}^{-2} \mathrm{~s}^{-1}\right]$, arrows indicates the direction of the flux. Panel (a) shows $\mathrm{H}^{+}$fluxes, panel (b) alpha particle fluxes and panel (c) heavy ion fluxes. Numbers in the figure (1 to 7) indicate regions for which sample distribution functions are shown in Fig. 3.

vertical and horizontal lines are shown in the lower panel, with black for the vertical line $\left(V_{x}=V_{\text {drift } x}\right)$ and red for the horizontal line $\left(V_{r}=V_{\mathrm{drift} r}\right)$. Solid lines show the fitted function, while circles connected with a solid line show measured data. As can be seen the fit is quite good except at the lowest energies. The low fluxes measured at the lowest energies are assumed to be caused by the limited measurement range rather than a true decrease for low energies. The relatively larger amount of ions just above measurement threshold could be due to a negative spacecraft charge accelerating cold plasma into the instrument, but a two-component plasma with a second lower temperature component can not be excluded. The low velocity part contribute relatively less to the flux than to the distribution function, so our fit is a good estimate of the total flux of the population partially measured by the instrument. The aver- 
age distributions, according to the fit, have temperatures in the range $3-10 \mathrm{eV}$.

\section{Results}

\subsection{Average flow around the planet}

Before we present the average distribution functions we will present the average flow around the planet calculated from these average distribution functions. The net flux in the $X$ and $R$ directions are calculated as for standard moment calculations (e.g. Fränz et al., 2006). The flux in the higher energy part is thus calculated by integrating the average distribution function, while the flux in the lower energy part is estimated using the fitting procedure described in the previous section. The flux in the two energy domains are added. What is obtained is therefore a net flux in the $X$ and $R$ directions, an isotropic distribution centered on zero would yield zero flux. Furthermore the $3 \mathrm{D}$ solid angle corresponding to each angular bin is used to calculate the flux, so it is a three-dimensional flux value which is obtained. Figure 2 shows the average flux of protons (panel a), alpha particles (panel b) and heavy ions (panel c) in cylindrical coordinates. Mars is indicated with a red-orange circle, units are Martian radii $\left(R_{\mathrm{M}}, 3393 \mathrm{~km}\right)$. White arrows indicate the flow direction, the colour scale shows the logarithm of the particle flux in units of $\mathrm{m}^{-2} \mathrm{~s}^{-1}$. The proton and alpha particles have been integrated for energies above $50 \mathrm{eV}$, for heavy ions we have integrated above $50 \mathrm{eV}$ and used a fitted Maxwellian below $50 \mathrm{eV}$. Black numbers indicate the position of the sample distribution functions (see next section). Zero flux is indicated with a low number, to distinguish it from no data which is shown as white. All spatial bins with less than $10^{4}$ energy spectrograms of data are treated as no data in Fig. 2. Each spatial bin contains 18 angular bins, which will have uneven sampling. With $10^{4}$ spectrograms there is on average of the order of $10^{3}$ spectrograms contributing to each angular bin which assures that we have good statistics. Lowering the threshold even down to $10^{2}$ just adds a few spatial bins and does not significantly change any results. The complete data coverage was shown in Nilsson et al. (2011).

A model induced magnetosphere boundary (IMB) and bow shock (Trotignon et al., 2006), derived using Phobos 2 and MGS data, is shown as a black line. It is known that the position of the induced magnetosphere boundary is highly variable (Brain et al., 2005; Trotignon et al., 2006; Edberg et al., 2009), so the average flux of protons and alpha particles should gradually decrease around the average induced magnetosphere boundary when moving closer to the planet. The opposite is true for planetary origin heavy ions. This is indeed what is seen in Fig. 2, the model is a good description of the dayside IMB, at least for the rather limited resolution we are using here. In the outer tail, beyond about $2 R_{\mathrm{M}}$ tailward, and at a radial distance of about $1.5 R_{\mathrm{M}}$ and outward, we see large fluxes of protons inside the model IMB. The planetary origin ions, while clearly present in the same region inside the IMB, has significantly smaller flux than closer to the center of the tail. To some extent this is because we show the flux density $\left(\mathrm{m}^{-2} \mathrm{~s}^{-1}\right)$ in Fig. 2, which decreases at larger radial distances as the ion flow is spread out over a larger area.
The flow direction of the solar wind ions is around the obstacle as can be expected. There is a significant amount of heavy ions outside the nominal induced magnetosphere boundary on the dayside of the planet. The flow direction of these ions is more in the $R_{\mathrm{MSO}}$ direction, with a relatively smaller component in the anti-sunward direction, as compared to the solar wind origin fluxes. The bow shock can be clearly seen in the $\mathrm{H}^{+}$and alpha particle data, as the region where the flux start to deviate around the obstacle. Two features are noteworthy concerning the magnetosheath flow. One is that the apparent bow shock, defined as the region where the flow start to deviate around the obstacle, is somewhat closer to the planet than the average bow shock position determined from wave data (Phobos 2) and electron and magnetic field data (MGS), i.e. as described in Trotignon et al. (2006). The difference is about one bin of $0.2 R_{\mathrm{M}}$ and is likely due to the different phase of the solar cycle for model and data. The flow direction of the heavy ion fluxes in the magnetosheath are well aligned with the model bow shock.

The other noteworthy feature of the magnetosheath ion flow is the distribution of alpha particles within the magnetosheath, which appears to be somewhat different from that of the protons. The alpha particle flux appears to be strongest in a plume along and just inside the bow shock, though the flow direction is not clearly aligned along the bow shock the way it is for the heavy ions.

Finally we note that the low proton fluxes in the solar wind, in the upper corner of the first panel of Fig. 2, may be due to poor statistics, but may also be due to an instrumental effect where narrow beams may be severely underestimated. This does not affect any of the conclusions drawn in the paper so we will not investigate this further.

\subsection{Average distribution functions}

For each spatial bin described in the previous section we have binned the measured energy spectrograms in angular bins, with the angle defined by the angle towards the sun (positive $X_{\mathrm{MSO}}$ ) and in the radial direction of the cylindrical coordinate system $R_{\mathrm{MSO}}$, with positive sign meaning away from the $x$ axis (center of the cylinder). This means that in practice we have calculated an average distribution function in each spatial bin. These can be used to study the evolution of ion distributions as the ions move away from Mars. We will present 7 such average distribution functions that help us understand the processes acting on the ions. First we will show four ion distributions from the tail, from the distant center of the tail to close to the planet. Then we will show two distributions from close to the terminator, one inside the nominal IMB, and one a significant distance outside. Finally we will show an ion distribution function from the dayside, just outside the nominal IMB. These are representative of the different regions where significant heating and acceleration have affected the ion distributions. Figure 3 shows 7 sample average distribution functions for protons, alpha particles and heavy ions (in the mass range of $\mathrm{O}^{+}$, $\mathrm{O}_{2}^{+}, \mathrm{CO}_{2}^{+}$). The samples are taken from the regions indicated with number 1 to 7 in Fig. 2. We have chosen to use differential flux for the colour scale rather than distribution function values, to emphasize high energy populations and provide a visual estimate of the flux. The coordinate system 
1

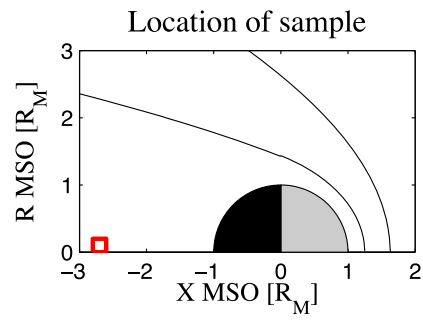

2

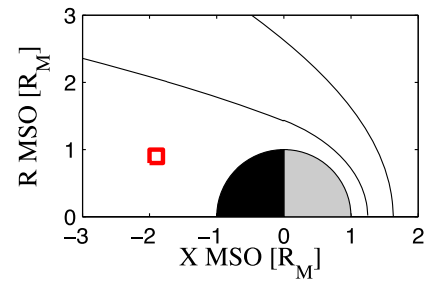

3

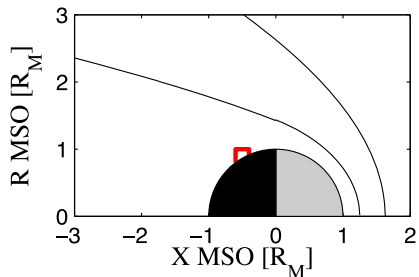

4

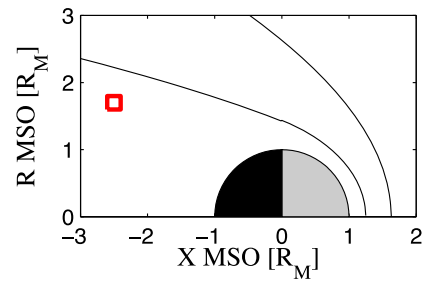

5
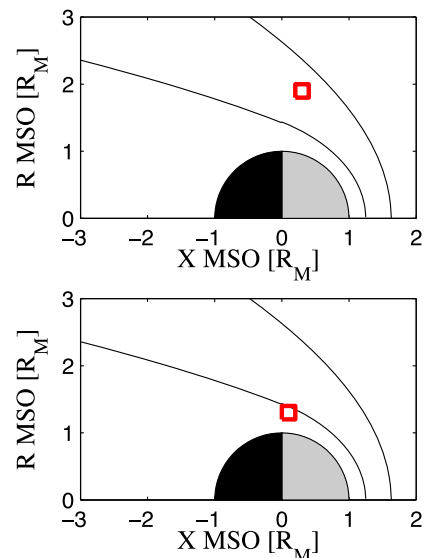

7

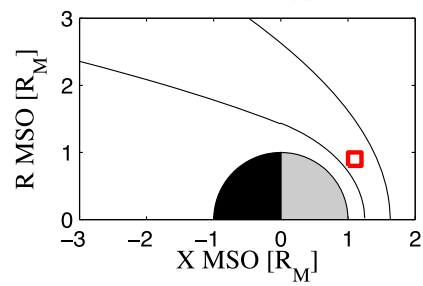

$\log _{10}\left[\mathrm{~m}^{-2} \mathrm{~s}^{-1} \mathrm{sr}^{-1} \mathrm{eV}^{-1}\right]$
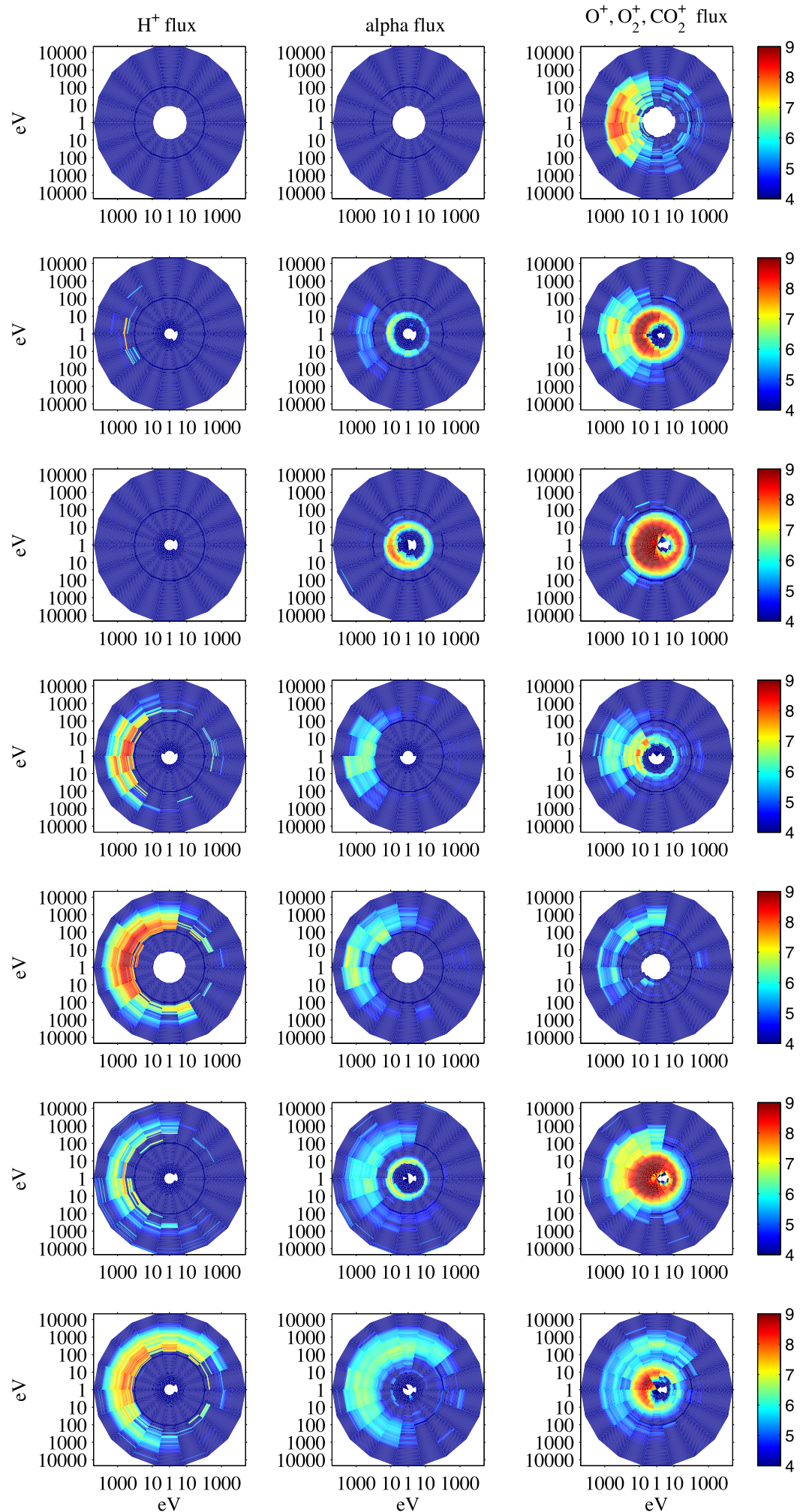

Fig. 3. Average distribution of flux for 7 selected regions in the near-Mars space, as indicated in Fig. 2. Column 1 shows the position where the sample data ware taken. Column 2 shows $\mathrm{H}^{+}$fluxes, column 3 alpha particle fluxes and column 4 show heavy ion fluxes $\left(\mathrm{O}^{+}, \mathrm{O}_{2}^{+}, \mathrm{CO}_{2}^{+}\right)$. The colour scale indicates the logarithm of the differential flux $\left[\mathrm{m}^{-2} \mathrm{~s}^{-1} \mathrm{sr}^{-1} \mathrm{eV}^{-1}\right]$, the $x$ and $y$ axis are logarithmically scaled in $\mathrm{eV}$, but with direction given by the $X_{\mathrm{MSO}} R_{\mathrm{MSO}}$ coordinate system used in Fig. 2, i.e. $+X$ towards the sun, $+R$ outwards from the $X$ axis, away from the planet.

of Fig. 3 is the same as used in Fig. 2, i.e. $+X$ towards the sun, $+R$ outwards from the $X$ axis, away from the planet. To aid in quantitative estimates of the energy levels, we provide line plots of the distributions, summed over all direc- tions, in Fig. 4. Blue lines shows $\mathrm{H}^{+}$fluxes, green lines alpha particle fluxes, and red lines show heavy ion fluxes $\left(\mathrm{O}^{+}, \mathrm{O}_{2}^{+}, \mathrm{CO}_{2}^{+}\right)$.

At point 1 , in the deep tail, we see no solar wind origin 

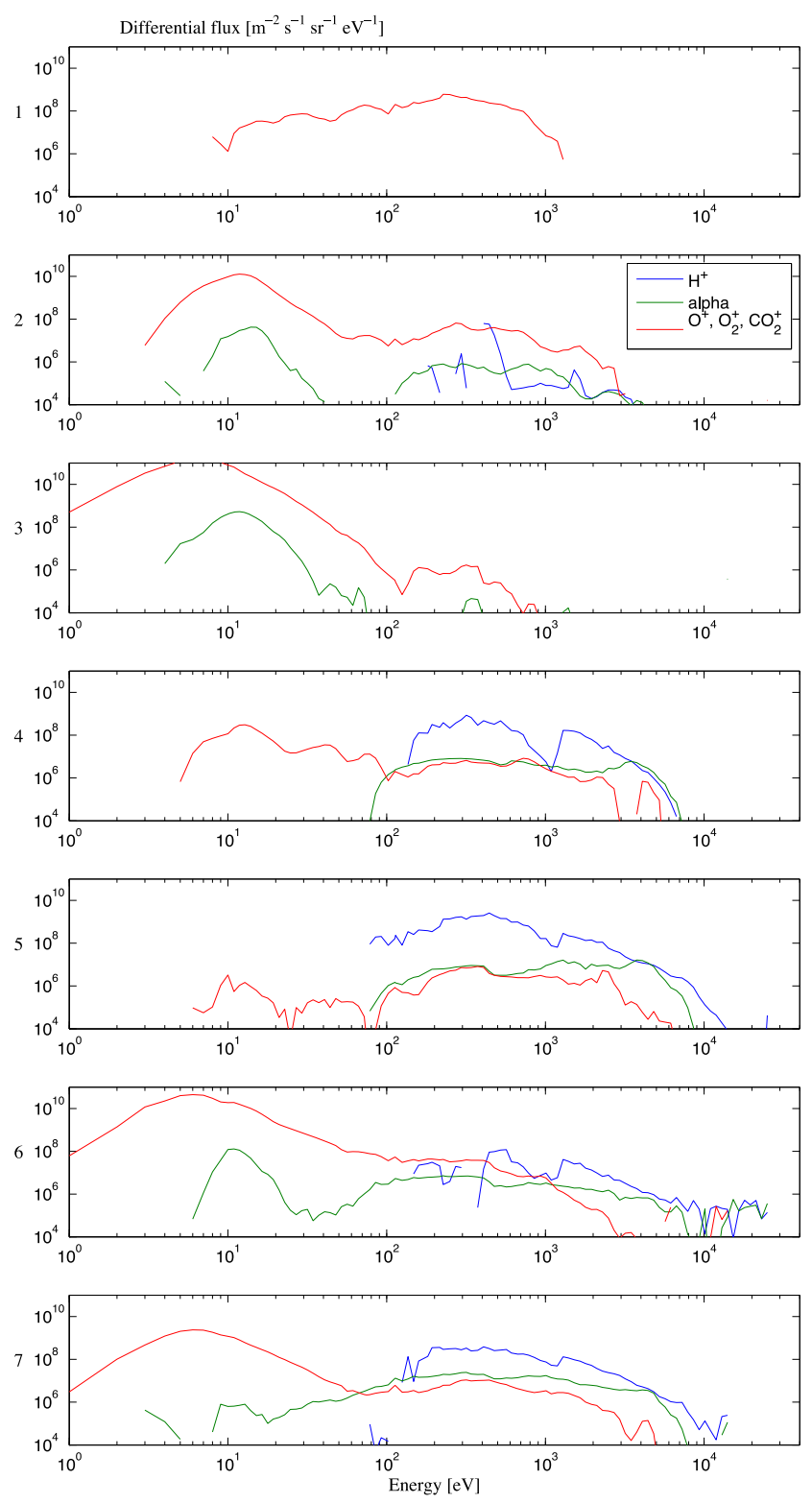

Fig. 4. Statistical distribution of flux for 7 selected regions in the near-Mars space, as indicated in Fig. 2. Blue lines shows $\mathrm{H}^{+}$fluxes, green lines alpha particle fluxes, and red lines show heavy ion fluxes $\left(\mathrm{O}^{+}, \mathrm{O}_{2}^{+}, \mathrm{CO}_{2}^{+}\right)$.

ions and most heavy ions have energies between 100 and $1000 \mathrm{eV}$. The heavy ions have a clear peak in their distribution for anti-sunward flow with only small fluxes in the sunward direction. Considering the long integration time the heavy ions mainly form tailward flowing beams with some deviations in the direction, consistent with previous reports Carlsson et al. (2006).

At point 2, somewhat closer to the planet and further away from the center of the tail, we see an added component of colder heavy ions, with only small fluxes of solar wind origin ions. The lower energy ions are mainly drifting tailward as well. Note that the unit used in the plot is flux, not phase space density. The distribution function is close to a tailward drifting Maxwellian. A detailed sample was discussed in Section 3.1. The temperature of the fitted Maxwellian distribution is $5 \mathrm{eV}$. The more energetic ions above about $100 \mathrm{eV}$ form tailward flowing beams. We do see some low energy ions at about $10 \mathrm{eV}$ energy in the alpha channel. Previous reports have attributed such low energy ions to $\mathrm{H}_{2}^{+}$ions (Lundin et al., 2009) rather than alpha particles.

Point 3 is located at a relatively low altitude in the nightside tail of Mars. We see more intense fluxes of low energy heavy ions, reaching down to almost $0 \mathrm{eV}$ (measured through ram-shift of the measured ions, the lowest energies are reached for the heaviest ions). Statistics are relatively poor for the lowest energies which can only be reached through the spacecraft ram energy shift, but still exhibits a quite smooth curve from 1 up to about $100 \mathrm{eV}$. The fitted temperature is $4 \mathrm{eV}$. The low energy component of the heavy ions is now dominating, but some accelerated ions are seen already here, with a rather random angular distribution. The flux of low energy ions detected in the alpha channel (mass per charge, $m / q$, of 2 ) is more intense than further tailward. Some of these particles are detected at more magnetosheath-like energies (best seen in Fig. 4). Whether these are $\mathrm{H}_{2}^{+}$ions that have been accelerated or intruding magnetosheath alpha particles cannot be directly resolved from the observations alone. However one should note that the more energetic part of these $m / q 2$ ions $(>50 \mathrm{eV})$ are the ones used to obtain Fig. 2, and these are flowing inward in the tail, as opposite to the heavy ions which are flowing straight anti-sunward or somewhat outward. This seems to indicate that the more energetic component in the alpha mass channel is indeed due to solar wind origin alpha particles penetrating into the tail. The flux of protons at magnetosheath energies is low.

At point 4 , located quite far downstream, but well away from the center of the tail, we see tailward flowing solar wind origin ions at the same time as heavy ions. The heavy ions are observed at energies from a few eV up to a few $\mathrm{keV}$. The fitted temperature is $8 \mathrm{eV}$. The flux in the higher energy part (above $100 \mathrm{eV}$ ) dominates, note that we show differential flux per eV in Fig. 4 and use a logarithmic scale for the energy. The heavy ions have a clear peak in their distribution for the tailward flow direction. The protons have a somewhat narrower energy distribution as compared to further upstream in the magnetosheath (point 5).

Point 5 is located outside the nominal induced magnetosphere boundary (IMB), somewhat sunward of the terminator. As expected we see tailward flowing solar wind origin ions of magnetosheath-like character, with energies between $100 \mathrm{eV}$ up to almost $10 \mathrm{keV}$. There are no significant heavy ion fluxes at the lowest measurable energies and the fluxes are relatively low below $100 \mathrm{eV}$. The heavy ions show a peak in their distribution in the radially outward direction. Energies of heavy ions reach a few $\mathrm{keV}$, much less than the highest energies of the solar wind origin ions. The shape of the average energy spectra are otherwise rather similar.

Point 6 is located at the terminator and just inside the nominal IMB. We do see magnetosheath-like fluxes of solar wind origin ions here, though the protons show weaker peak fluxes, as can be expected inside the nominal IMB. The heavy ions show much enhanced densities of a heated thermal population, i.e. fairly symmetric in angular space. 
The temperature of the fitted distribution is $5 \mathrm{eV}$. At energies above $100 \mathrm{eV}$ there is an enhancement of the fluxes in the tailward, radially outward direction. The alpha particle energy of the main, magnetosheath origin population extends down below $100 \mathrm{eV}$, whereas we once again see a clear local peak at about $10 \mathrm{eV}$, likely representing $\mathrm{H}_{2}^{+}$.

Point 7 is located in the front-side magnetosheath at a significant radial distance from the subsolar point and just outside the nominal IMB. This is a region where the integrated flow of the heavy ions is more radial than the flow direction of the solar wind origin ions (Fig. 2). The distribution of the heavy ions have a peak in the radially outward direction, close to perpendicular to the sun-planet line. The peak of the proton and alpha distributions are flowing around the planetary obstacle, having a mean flux with an angle of about $45^{\circ}$ outward from the anti-sunward direction. Low energy heavy ions are also observed this close to the nominal IMB, with a fitted temperature of $7 \mathrm{eV}$. Part of the heavy ion flow is directed towards the planet and could cause heavy ion sputter of the atmosphere of Mars. We have therefore made a first rough estimate of the flux in the lowest altitude dayside bins which is directed towards the atmosphere (taken as $\pm 60^{\circ}$ from the tailward direction summed over the lowest altitude dayside bins with radial position $R_{\mathrm{MSO}}<1 R_{\mathrm{M}}$ ). This flux is $0.4 \cdot 10^{23} \mathrm{~s}^{-1}$ for our average solar wind conditions.

4.3 Co-occurrence of heavy and solar wind origin ions With the average distribution functions it is not possible to tell if solar wind and planetary origin ions are present in the same statistical bin at the same time. This can be done using ordinary moment data, even though these may suffer from biased and limited sampling. Using moment data when at least either heavy ion flux or proton flux was nonzero, we can see how often the two populations are non-zero simultaneously in the same spatial bin. We have investigated the outer tail boundary which may be a superposition of a flapping tail, or show a region of real plasma mixing. We have done the same investigation for the magnetosheath, where we have investigated the region outside the nominal IMB sunward of $X=-1$.

For the outer tail region, inside the nominal IMB at $\left(X_{\mathrm{MSO}}<-2,1.5<R_{\mathrm{MSO}}<\mathrm{IMB}\right)$ the heavy ion flux and proton flux were non-zero simultaneously in $32 \%$ of the cases, only heavy ions were present in $23 \%$ of the cases, and only protons in $45 \%$ of the cases. In the nominal magnetosheath, sunward of $X_{\text {MSO }}=-1$, the heavy ion flux and the proton flux were non-zero in $37 \%$ of the cases, only heavy ions were present in $5 \%$ of the cases and only protons in $57 \%$ of the cases. Almost the same result is obtained for the nominal magnetosheath tailward of $X_{\mathrm{MSO}}=-1$. One should note that the final step of the data cleaning procedure, checking for apparent $\mathrm{O}^{2+}$ fluxes in the magnetosheath, was performed at the moment data level in this case. The time resolution of the moment calculation is only $192 \mathrm{~s}$. This is a coarser method than the one used otherwise in this paper, as it will remove all data at all energies during one time period of $192 \mathrm{~s}$ if a part of it is contaminated. Still we get a quite clear picture from the moment data. Heavy ions and protons are observed simultaneously as often in the outer tail region as in the nominal magnetosheath sun- ward of $X_{\mathrm{MSO}}=-1$. Thus we cannot really distinguish between tail flapping and a statistical IMB closer to the tail. An average fit in radial coordinates would clearly yield an IMB, based on ion observations, closer to the planet than the nominal IMB we use for comparison. The low occurrence of only heavy ions in the nominal magnetosheath indicates that the IMB model we are using is at least not overestimating the altitude of the IMB for the low solar activity conditions prevailing during our observations. Some observations of heavy ions only should be expected outside the model average IMB, as the IMB is dynamic.

\subsection{Acceleration of ions in the tail and the total flux in the tail}

Moment calculations of the ion velocity in the tail show a gradual acceleration of the ions with tailward distance (Nilsson et al., 2010). Lundin et al. (2008b) divided the data into energy ranges below and above $200 \mathrm{eV}$. They showed that the lower energy population decreased with tailward distance, and the higher energy population increased. Using the average distribution functions we can investigate this process in more detail. Figure 5 shows 10 energy spectra (summed over all angles) from 12 distances in the tail, from $X_{\mathrm{MSO}}$ values of -2.8 to $-0.6 R_{\mathrm{M}}$, one bin $\left(0.2 R_{\mathrm{M}}\right)$ wide, and summed over radial distances up to $1 R_{\mathrm{M}}$. Spectrograms at all distances show two peaks, one at low energy (about $10 \mathrm{eV}$ ) and one peak between about 100 and about $2000 \mathrm{eV}$. A thick black line shows the energy spectrogram obtained in the most tailward part, a thick grey line the spectrogram from closest to the planet. The flux in the low energy population decreases, and the flux in the high energy population increases with tailward distance. The low energy population is somewhat shifted to higher energies for higher tailward distance. The higher energy population does show some increase in the observed energy range with tail distance, but tailward of about $-1 R_{\mathrm{M}}$ there is not much change in the

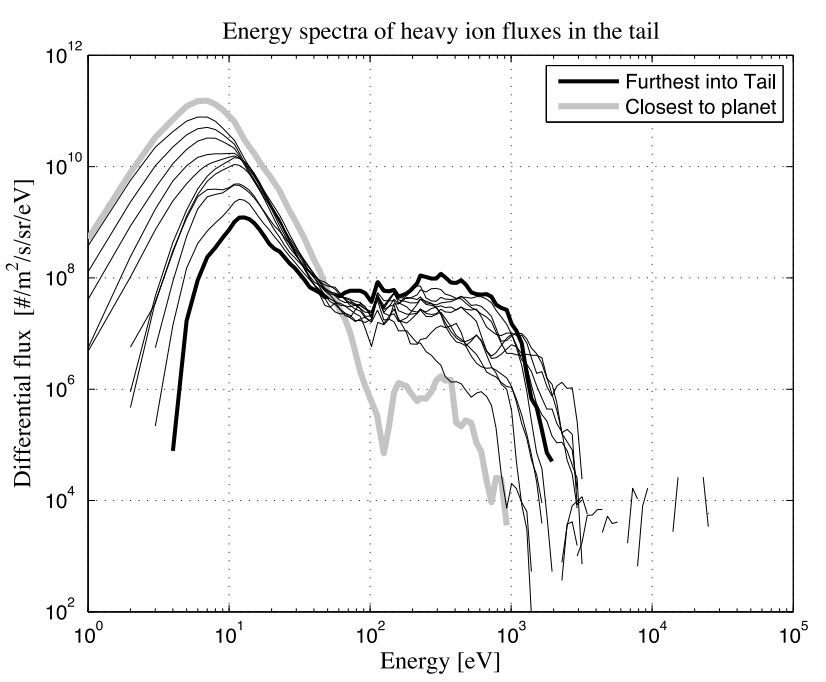

Fig. 5. Energy spectra from the magnetotail, at $X_{\text {MSO }}$ positions between -2.8 and $-0.6 R_{\mathrm{M}}$, summed over a region one bin $\left(0.2 R_{\mathrm{M}}\right)$ wide in the $X$ direction, and summed over radial distances up to $1 R_{\mathrm{M}}$. The $x$ axis shows energy [eV] and the $y$ axis shows differential flux $\left[\mathrm{m}^{-2} \mathrm{~s}^{-1} \mathrm{sr}^{-1}\right.$ $\left.\mathrm{s}^{-1}\right]$. The thick black line shows the energy spectrogram obtained in the most tailward part, the thick grey line the spectrogram from closest to the planet. 


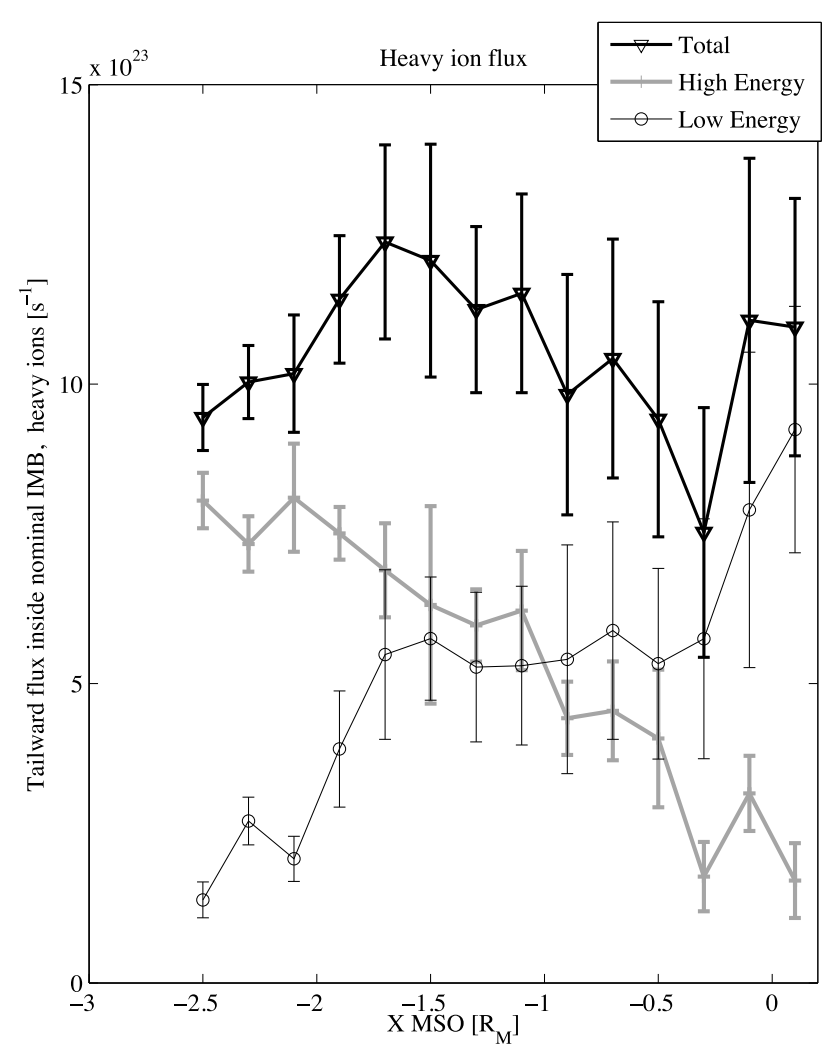

Fig. 6. The total tailward heavy ion flux inside the nominal IMB $\left[\mathrm{s}^{-1}\right]$ as function of tail distance $\left[R_{\mathrm{M}}\right]$ is shown with a bold black line and triangles. Total flux from the low energy component (fit to ions below $50 \mathrm{eV}$ ) is shown with a thin black line with circles. The total flux in the high energy component (above $50 \mathrm{eV}$ ) is shown with a thick grey line with crosses. Error bars indicate the standard deviation of the total flux of all angular bins added as independent variables.

shape of the average energy distribution. The total flux remains approximately constant. Due to the logarithmic scale of Fig. 5 this is difficult to see in the figure, and the total flux is also dependent on the total cross sectional area of the tail which increase with tailward distance. Therefore we show the total tailward flux inside the IMB in Fig. 6. The total flux is shown with a bold black line with triangles. The low energy component $(<50 \mathrm{eV})$ is shown with a thin black line with circles, and the high energy component $(>50 \mathrm{eV})$ is shown with a thick grey line. Error bars indicates the standard deviation of the total flux of all angular bins, added as independent variables. Despite the shift in the energy range, from dominating low energy component at the terminator to almost all in the high energy component in the deep tail, the total flux remains approximately constant, as it should if both components are properly estimated. There is one notable dip in the total flux, at $X_{\text {MSO }}=-0.3$. It occurs in a region in the nightside/near tail with poor spatial coverage (see Fig. 2). This increases the uncertainty more than indicated by the standard deviation, which does not include uncertainties due to poor spatial coverage.

The low energy component is estimated from the Maxwellian fit described in Section 3.1. The good continuity with tail distance of the total flux, despite the acceleration of the ions in the tail, thus indicate that our low energy flux estimates at the terminator is a good estimate of the to- tal flux. The expected continuity is based on the assumption that the trans-terminator flow is the main source of ions in the tail, and that the lack of data points in the planet shadow thus does not strongly influence our results. If they did, we should see a significant increase in the tail fluxes as the data coverage improve with tailward distance. This is not the case, though we do see a dip at $X_{\mathrm{MSO}}=-0.3$. This indicates that most of the tail flux passes through the region we can observe at $X_{\mathrm{MSO}}=-1$, and that the tailward transterminator flux equals the total tail escape flux. The total escape through the tail, and thus the trans-terminator flux, estimated from our data set has been reported by Nilsson et al. (2011). The average tail escape flux inside the nominal IMB is $1.1 \cdot 10^{24} \mathrm{~s}^{-1}$, of a total escape of $2 \pm 0.2 \cdot 10^{24} \mathrm{~s}^{-1}$. 4.5 Acceleration of heavy ions during CIR events

If more energy is put into the Mars ionosphere this may lead to increased escape of planetary ions, as has been shown for our data set by Nilsson et al. (2011). For modeling of the ion escape, it is important to know how much of the increased energy input goes into more escaping particles, and how much goes into further acceleration of particles that would anyway escape. CIR events represents a pronounced increase in the energy flux in the solar wind and are therefore very suitable to study the response of the near Mars space to increased energy input from the solar wind. We have used the same CIR events as in Edberg et al. (2010) to make a data set which can be compared with the average conditions (excluding the CIR events). The CIR data consists of 41 high pressure events observed between July 2007 and September 2008.

Figure 7 shows heavy ion average energy spectra obtained in the tail (upper panel) and in the dayside magnetosheath (lower panel), for average conditions (black line) and during CIR events (red line). The dayside magnetosheath data is taken from a region 5 bins wide in the $R_{\mathrm{MSO}}$ direction, starting from $0.4 R_{\mathrm{M}}$ outside the nominal IMB, for $X_{\mathrm{MSO}}$ distances between 0 and $1 R_{\mathrm{M}}$. The tail energy spectra are averaged for $X_{\mathrm{MSO}}$ distances between -2.8 and $-1 R_{\mathrm{M}}$, and inside a radial distance of $1 R_{\mathrm{M}}$. There is no significant cold population in the magnetosheath, and the shape of the energy distributions during CIR events and during average conditions are quite similar. Furthermore a local decrease in the heavy ion fluxes in the magnetosheath can be seen between $500 \mathrm{eV}$ and about $1 \mathrm{keV}$. This is the region where protons may contaminate the heavy ion flux estimates. When such contamination happens the algorithm assumes that all counts in the heavy ion channels are due to contaminating protons. The decrease of the heavy ion flux in this energy range indicates that this leads to an underestimate of the heavy ion flux in this particular energy range.

The shape of the tail distributions during CIR events and during average conditions are also quite similar, with only a slight shift of the higher energy population in the tail towards higher energy during CIR events.

\section{Discussion}

\subsection{Distribution functions}

Three features of the average distribution functions are important. The first is that the average low energy populations are close to Maxwellian, and whereas the bulk drift 

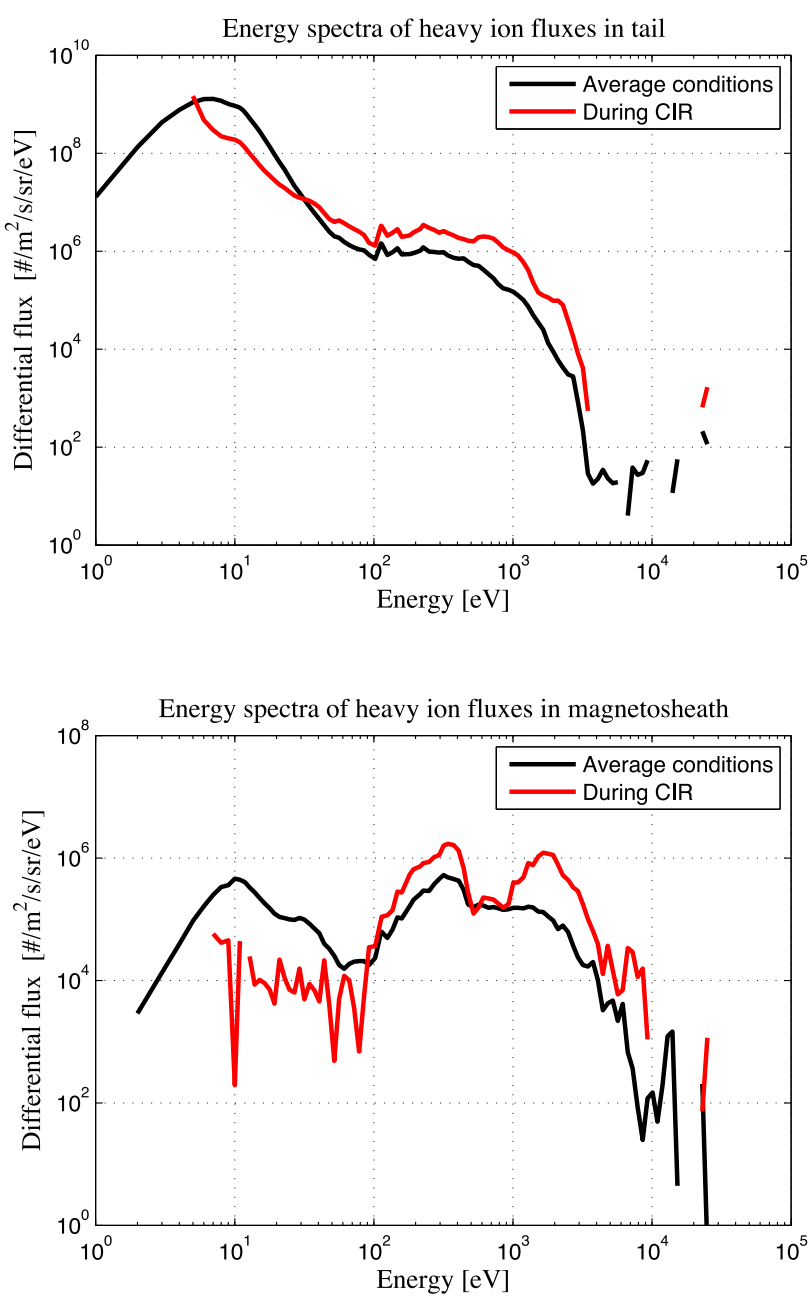

Fig. 7. Average heavy ion spectra from the tail (upper panel) and from the dayside magnetosheath (lower panel). Spectra for average conditions are shown with black lines, and during CIR events are shown with red lines. The $x$ axis shows particle energy summed over all angles [eV], the $y$ axis shows the differential flux $\left[\mathrm{m}^{-2} \mathrm{~s}^{-1} \mathrm{sr}^{-1} \mathrm{~s}^{-1}\right]$.

has a tailward component, the distributions show significant fluxes of sunward moving ions as well (i.e. see the sample distribution of Fig. 1 and examples 2, 3 and 6 of Fig. 3 for clear cases). The altitudes where these ions are observed are in the non-collisional regime (the exobase is expected to be at $195-215 \mathrm{~km}$ altitude). Gyration in a background magnetic field and wave-particle interaction reasonably give rise to the thermal populations with particles moving in all directions. In the energy range of a few $\mathrm{eV}$ gravity may also play a role, as the escape energy for heavy ions at Mars is 2 to $5 \mathrm{eV}$. Wave-particle interaction at Mars has previously been discussed by Ergun et al. (2006). Our data confirm the importance of heating processes in the upper ionosphere of Mars. Due to lack of wave measurements we cannot directly study the heating mechanism. We do note that in the upper magnetosphere of Earth, where background magnetic fields are of the same order of magnitude as in the induced magnetosphere of Mars, outflowing heavy ions are effectively heated by what appear to be Alfvén waves (Nilsson et al., 2006b; Slapak et al., 2011; Waara et al., 2011).

The outflowing ions at Mars are accelerated to form tailward flowing beams, apparently at least in part by the solar wind motional electric field (Dubinin et al., 2006; Fedorov et al., 2006; Nilsson et al., 2010). The second important feature of the average distribution functions is that the tailward flowing beams show a distinct distribution in energy space, in most of the tail well separated in energy from the low energy ions. See Fig. 5 for a clear distinction between the two energy populations, see sample distributions 1, 2 and 4 of Fig. 3 for two-dimensional samples. Further tailward, the flux in the average low energy population decreases and the flux in the average high energy population increases. The general increase of the higher energy population with distance from the terminator implies that acceleration occur throughout the tail. Tailward of $X_{\mathrm{MSO}}=-1 R_{\mathrm{M}}$ the two populations show rather small variations in the average shape of their energy distributions and seem to remain distinct from each other. This suggests that acceleration occur quickly in a relatively confined region. If acceleration is fast/confined in space, ions are more likely observed before or after acceleration. The further tailward, the more likely that the ions have already been accelerated at the time of observation. Our conclusion is that acceleration occur throughout the tail, but in a confined region in the $Y_{\mathrm{MSO}^{-}}$ $Z_{\text {MSO }}$ plane. Different types of acceleration in the Martian tail was discussed by Fedorov et al. (2006), where acceleration by the solar wind electric field and $j \times B$ tension forces are likely alternatives. Heating and subsequent tailward acceleration by the mirror force can also be a viable explanation depending on the geometry of the draped fieldlines.

The third important feature relates to the heavy ions observed in the magnetosheath. These have a stronger $R_{\mathrm{MSO}}$ component than the solar wind origin flow. They also have a rather low energy as compared to classical solar wind pick up ions, at or below the energy of the solar wind origin specie. Finally, they do not form torus or shell distributions. Pick up ions will upon creation move in the direction of the magnetosheath electric field which is consistent with the radial flow direction. In due time, in a constant background magnetic field, the picked up ions will gyrate and come back to near the starting point. If the ions could form a proper ring distribution this should yield a rather small net radial flow, consistent with the background magnetosheath flow. The gyro radius of energized heavy ions in the magnetosheath of Mars is on the order of a few hundred to a few thousand $\mathrm{km}$. A possible explanation for the observed near radial bulk flow is that the gyro radii of the ions we observe are of the same order of magnitude as the thickness of the magnetosheath close to the planet. Therefore the ions do not form well behaved torus distributions. Furthermore the electric and magnetic fields in the magnetosheath are variable on the time scale of a heavy ion gyration period which is of the order of 10 to $100 \mathrm{~s}$. A plume of ions extending in the solar wind electric field direction is a feature of a number of simulations (see Brain et al. (2010) for a summary and comparison of most recent Mars models). For the data set we are using here, taken after the operations of the Mars Global Surveyor spacecraft ceased, there is no local (Mars orbit) proxy available for the solar wind electric field direction. We can confirm the presence of a plume of heavy ions extending into the magnetosheath, but cannot investi- 
gate the dependence on the solar wind electric field direction. Previous work by Dubinin et al. (2006) have shown how ionospheric ions can be gradually accelerated by what appear to be a penetrating solar wind electric field. This means that the ions must have a significant motion in the direction of the electric field when they are accelerated. Our data is thus consistent with an ionospheric origin also for the magnetosheath heavy ions. It is therefore not clear from our data wether the magnetosheath heavy ions were created within the magnetosheath (pick up ions) or if they represent another escape path of ionospheric origin plasma. A careful examination of the mass of the escaping magnetosheath heavy ions could be used to distinguish between these possibilities, as exosphere pick up should be dominated by $\mathrm{O}^{+}$. It is noteworthy that the range of energies observed and the shape of the angular distribution (but not direction) are very similar for the accelerated magnetosheath and the accelerated tail heavy ion fluxes. The range of mass channels covered by the heavy ion species in the magnetosheath and tail are quite similar, suggesting an ionospheric origin for both populations. As noted earlier mass separation is less reliable at the energies observed in the magnetosheath, so we leave a more careful composition analysis for a future study.

We can confirm the presence of anti-sunward moving heavy ions in the dayside Mars magnetosheath and magnetosphere, which may precipitate and cause ion sputtering of the ionosphere. Sample data was shown in Fig. 3, sample 7. A rough estimate gives an approximate anti-sunward flux of $0.4 \cdot 10^{23} \mathrm{~s}^{-1}$ for our average solar wind conditions (see description at the end of Section 4.2). This must be considered as an upper limit, as higher resolution measurements, preferably binned in altitude and solar zenith angle, should be used to get a more reliable estimate of how much flux will really reach the exobase. We are currently working on such a data set.

Finally one may note the lack of significant sunward flowing ions in the magnetotail. Some models have predicted rather different distributions in the magnetotail of Mars, e.g. Fang et al. (2008). At our sample point 3 the rather weak accelerated fluxes seen could in part be made up of picked up ions that have moved back into the tail, so the phenomenon discussed by Fang et al. (2008) may indeed exist, but with a very different spatial distribution as compared to their model results. Modelling distribution functions, which can be compared with the observations presented here, will be an important step forward in understanding the ion dynamics at Mars.

\subsection{Dynamics and mixing of ion populations}

In Section 4.3 we showed that the outer tail region, inside the nominal IMB but with significant fluxes of solar wind origin ions, had somewhat lower co-occurrence of heavy and solar wind origin ions than the magnetosheath. Occurrence of $\mathrm{O}^{+}$only, without any solar wind origin ions, was much more common than outside the nominal IMB, as can be expected. This support the picture of a moving tail boundary as an explanation for the outer tail region with mixed ion populations, as opposite to a region with mixing of the two regions as a result of boundary instabilities. In the latter case we would expect higher co-occurrence of heavy and solar wind ions in the mixing region as compared to in the magnetosheath.

It is noteworthy that significant fluxes of solar wind origin ions were present inside the nominal IMB in the outer tail, tailward of $X=-2$, in the majority of all cases. Solar wind origin ions were present in $77 \%$ of the cases, with solar wind origin ions only in $45 \%$ of the cases. Therefore the nominal IMB of the Trotignon et al. (2006) model appears incorrect tailward of $X=-2$ for solar minimum conditions. With solar wind origin ion fluxes going from low at $R=1.6$ to high at $R=2$, putting the average tail boundary at $R=1.8$ would give a better estimate than the IMB model we have been using. Agreement is better with the fit of MGS data provided by Vignes et al. (2000) which have an approximately constant radial distance of the tail IMB tailward of $X=-2$.

We suggest that the tail IMB should be studied based on ion data using boundary identifications on an orbit by orbit basis in order to improve these initial results.

The low occurrence of heavy ions only, no solar wind origin ions, in the nominal magnetosheath indicates that the IMB model of Trotignon et al. (2006) is at least not over-estimating the altitude of the IMB sunward of $X=$ -2 . Visual inspection of Fig. 2 indicates that the model is accurate for this region within the accuracy of the binning used in our study. The study of Edberg et al. (2009) showed that for the conditions between 2004 and 2009 the IMB (magnetic pile-up boundary when determined from MGS data) at the terminator varied about $0.2 R_{\mathrm{M}}$ with solar EUV changes, and a similar amount between no crustal fields and the strongest crustal fields. Not taking crustal magnetic fields into account will thus smear our boundary up to one data bin at the terminator, less in the dayside. A similar smear will occur for position changes due to the changing solar cycle.

Another dynamic region in space around Mars is the bow shock. The average position is clearly seen in our data as a change in the flow direction from anti-sunward to around the planetary obstacle. The significant change of the average flow direction occurs well inside the statistical bow shock crossing determined from plasma waves data (Phobos 2) and magnetic field and electron data (MGS spacecraft). The discrepancy between our bow shock determined using the solar wind origin ion flow direction and the Trotignon et al. (2006) model is likely because our measurements were made during the minimum of a solar cycle (2007-2010), whereas the model is based on data from solar maximum (Phobos) and the increasing phase of a solar cycle. Edberg et al. (2009) have shown that the bow shock position is sensitive to the solar EUV flux which varies with solar cycle. The variation in the average bow shock position at the terminator was $0.4 R_{\mathrm{M}}$ for the range of solar EUV flux obtained between 2004 and 2009. This is consistent with our observed difference between the Trotignon et al. (2006) bow shock position and the deviation of the ion flow from anti-sunward of about $0.2 R_{\mathrm{M}}$.

The flux of heavy ions in the dayside magnetosheath is well aligned with the model bow shock, and significant heavy ion fluxes occur approximately out to the model bow shock. Heavy ion fluxes thus occur somewhat further out than the average position of the bow shock for the solar 
conditions of the observations. Heavy ion fluxes are thus present in the bow shock region at Mars, and could possibly affect the physics of the bow shock region. Yamauchi et al. (2011) have shown that the bow shock region at Mars is different from that at Venus and Earth. Clearly a study should be made of simultaneous bow shock signatures in electron, solar wind and heavy ion data.

Another feature of our data is that the alpha particle fluxes have a somewhat different distribution than the protons. The alpha particle fluxes are enhanced in a plume along the bow shock. This is a type of signature that could occur in multi species models, and is worthwhile to look for in simulation results.

\subsection{Trans-terminator flow}

We showed that our estimates of the tailward total heavy ion flux inside the IMB remained close to constant with tailward distance, despite the shift from a mixture of the cold and accelerated populations close to the planet and a dominating accelerated component deeper into the tail. This support our escape flux estimates for low energy ions obtained close to the planet and reported in Nilsson et al. (2011). Our estimates are an order of magnitude lower than the transterminator fluxes reported by Fränz et al. (2010), who based their estimates on careful fitting of theoretical distribution functions to 7 particularly suitable orbits. The discrepancy between our result and that of Fränz et al. (2010) can be explained in three different ways:

(1) Their 7 cases are not typical. Ionospheric origin ion fluxes are known to show large variations at Mars. These variations may also be spatial, which leads to the second possibility.

(2) The trans-terminator flux is not uniform, as assumed when the single point observations of Fränz et al. (2010) are extrapolated to give a total flux. With our method, fluxes may be non-uniform as long as they are sampled in a symmetric fashion. Possible sources of asymmetry which could affect our estimate include uneven sampling of the northern and southern hemisphere, and uneven sampling relative to the direction of the solar wind electric field. In Lundin et al. (2011) it was shown that the tail fluxes show no hemispheric asymmetry, so this would not affect our results. Nilsson et al. (2011) showed that there are flow asymmetries with more escape from the north and dusk as compared to south and dawn. The average result obtained when calculating escape using these for quadrants or ordinary cylinder symmetry was however negligible. The solar wind electric field direction depends on the direction of the solar wind magnetic field, which alternates depending on whether Mars is above or below the heliospheric current sheet. For shorter periods of time, this could be a problem, especially for a few case studies. For almost four years of data the solar wind electric field will have an even distribution, with the positive and negative "electric field hemispheres" sampled equally often.

(3) There is a cold population also in the tail, which is not measured by the particle detector and not well approximated by our Maxwellian fit. Spacecraft charging and limited energy resolution may indeed prevent observations of a low energy population, so this is a possibility. However the continuity of our tail flux estimates with tail distance, despite the increasing dominance of the accelerated population, does indicate that our low energy flux estimates close to the planet yield a good estimate of the average escape flux, consistent with the higher energy measurements obtained deeper into the tail.

\section{Conclusions}

We have shown average distribution functions of solar wind origin and heavy ionospheric origin ions in the vicinity of Mars. These show clear evidence of a heated ionospheric ion population as the main source of the escaping ion fluxes in the tail of Mars. Our results show that the gradual increase of the average bulk speed in the tail is primarily due to a shift between a cold population at energies of about $10 \mathrm{eV}$, which dominates close to the planet, and an accelerated population with energy typically in the range from $100 \mathrm{eV}$ to $2000 \mathrm{eV}$, which appear to get most of its acceleration in a confined region. No significant fluxes of planetward moving ions are seen in the tail.

Our data set shows a very good continuity in the estimated total tailward flux inside the IMB. This suggests that the nightside ionosphere, not covered by our measurements is not a strong source (as can be expected). Furthermore this indicates that the average total trans-terminator flux is an order of magnitude lower than the estimates obtained by Fränz et al. (2010) based on a few carefully selected and studied events.

The ionosphere may also be the source for the heavy ion fluxes from the dayside and terminator regions through the magnetosheath, although classical pick up of exospheric atoms and molecules ionized inside the magnetosheath may also be a viable source. Careful modeling of the resulting ion distributions should be able to distinguish between these scenarios through comparison with our observations. The heavy ion magnetosheath fluxes form a plume extending from the dayside ionosphere and terminator region, along the bow shock. The flow direction is more radial, approximately along the bow shock, than the solar wind origin ion fluxes in the magnetosheath.

We can confirm the presence of heavy ions moving towards the planet in the dayside magnetosphere and magnetosheath. The total planetward flux in our lowest altitude bins (covering altitudes from the minimum $275 \mathrm{~km}$ up to $0.2 R_{\mathrm{M}}=680 \mathrm{~km}$ ) is $0.4 \cdot 10^{23} \mathrm{~s}^{-1}$, which should be considered an upper limit for the flux that actually reaches the exobase.

Our comparison of the average energy spectra during average solar wind conditions and during CIR events indicates that the average ion energy is not strongly affected by the CIR. This means that the increase in available energy is relatively effective in removing ions, as not much energy is wasted in further acceleration of ions that would anyway escape.

Our observations in the outer tail beyond an $X$ distance of $-2 R_{\mathrm{M}}$ indicate that the IMB model of Trotignon et al. (2006) is not good for predictions of where to find solar 
wind origin and ionospheric origin ions for low solar activity. It appears as the average tail IMB should be narrower, a straight line at a radial distance of about $1.8 R_{\mathrm{M}}$ seems suitable, although identification of the IMB position on an orbit by orbit basis should be used for an improved tail IMB model based on ion data. Finally we suggest that the heavy ion fluxes in the dayside magnetosheath affect the Martian bow shock. A detailed comparison of the bow shock as determined from electron data (as for the MGS data) and from ion data (as in our data set) should be performed. The bow shock as based on ion data during low solar cycle is closer to the planet than in the Trotignon et al. (2006) model, which is consistent with the solar EUV dependence of the position of the bow shock determined by Edberg et al. (2009).

Acknowledgments. The work was supported by the Swedish National Space Board. The work of HN and NJTE was supported by the Swedish Research Council. The work of GS was supported by the National Graduate School of Space Technology, Luleå University of Technology.

Guest editor J. S. Halekas thanks two anonymous reviewers for their assistance in evaluating this paper.

\section{References}

Acuña, M. H., J. E. P. Connerney, P. Wasilewski, R. P. Lin, K. A. Anderson, C. W. Carlson, J. McFadden, D. W. Curtis, D. Mitchell, H. Rème, C. Mazelle, J.-A. Sauvaud, C. d'Uston, S. J. Bauer, P. Cloutier, M. Mayhew, D. Winterhalter, and N. F. Ness, Magnetic field and plasma observations at Mars: Initial results of the Mars Global Surevor mission, Science, 279, 1676-1680, doi:10.1126/science.279.5357.1676, 1998.

Barabash, S. and the ASPERA-3 team, The Analyzer of Space Plasmas and Energetic Atoms (ASPERA-3) for the Mars Express mission, Space Sci. Rev., 126, 113-164, 2006.

Barabash, S., A. Fedorov, R. Lundin, and J.-A. Sauvaud, Martian atmospheric erosion rates, Science, 315, 501-503, 2007.

Brain, D., J. S. Halekas, R. Lillis, D. Mitchell, R. Lin, and D. Crider, Variability of the altitude of the Martian sheath, Geophys. Res. Lett., 32, doi:10.1029/2005GL023126, 2005.

Brain, D., S. Barabash, A. Boesswetter, S. Bougher, S. Brecht, G. Chanteur, D. Hurley, E. Dubinin, X. Fang, M. Fraenz, J. Halekas, E. Harnett, M. Holmstrom, E. Kallio, H. Lammer, S. Ledvina, M. Liemohn, K. Liu, J. Luhmann, Y. Ma, R. Modolo, A. Nagy, U. Motschmann, H. Nilsson, H. Shinagawa, S. Simon, and N. Terada, A comparison of global models for the solar wind interaction with Mars, Icarus, 206, 139-151, doi:10.1016/j.icarus.2009.06.030, 2010.

Carlsson, E., A. Fedorov, S. Barabash, E. Budnik, A. Grigoriev, H. Gunell, H. Nilsson, J.-A. Sauvaud, R. Lundin, Y. Futaana, H. Holmström, H. Andersson, M. Yamauchi, D. Winningham, R. Frahm, J. Sharber, J. Scherrer, A. Coates, D. R. Linder, D. O. Kataria, E. Kallio, H. Koskinen, T. Säles, P. Riihilä, W. Schmidt, J. Kozyra, J. Luhmann, E. Roelof, D. Williams, S. Livi, C. Curtis, K. C. Hsieh, B. R. Sandel, M. Grande, M. Carter, J.-J. Thocaven, S. McKenna-Lawlor, S. Orsini, R. CerulliIrelli, M. Maggi, P. Wurz, P. Bochsler, N. Krupp, J. Woch, M. Fraenz, K. Asamura, and C. Dierker, Mass composition of the escaping plasma at Mars, Icarus, 182, 320-328, 2006

Connerney, J. E. P., M. H. Acuna, P. J. Wasilewski, G. Kletetschka, N. F. Ness, H. Réme, R. P. Lin, and D. L. Mitchell, The global magnetic field of Mars and its implications for crustal evolution, Geophys. Res. Lett., 28, 4015-4018, 2001.

Dubinin, E., R. Lundin, M. Fränz, J. Woch, S. Barabash, A. Fedorov, D. Winningham, N. Krupp, J.-A. Sauvaud, M. Holmström, H. Andersson, and M. Yamauchi, Electric fields within the martian magnetosphere and ion extraction: ASPERA-3 observations, Icarus, 182, 337$342,2006$.

Edberg, N. J. T., M. Lester, S. W. H. Cowley, and A. I. Eriksson, Statistical analysis of the location of the Martian magnetic pileup boundary and bow shock and the influence of crustal magnetic fields, J. Geophys. Res., 113, A08206, doi:10.1029/2008JA013096, 2008.

Edberg, N. J. T., D. A. Brain, M. Lester, S. W. H. Cowley, R. Modolo, M. Fränz, and S. Barabash, Plasma boundary variability at Mars as observed by Mars Global Surveyor and Mars Express, Ann. Geophys.,
27, 3537-3550, doi:10.5194/angeo-27-3537-2009, 2009.

Edberg, N. J. T., H. Nilsson, A. O. Williams, M. Lester, S. E. Milan, S. W. H. Cowley, M. Fränz, S. Barabash, and Y. Futaana, Pumping out the atmosphere of Mars through solar wind pressure pulses, Geophys. Res. Lett., 37, L03107, doi:10.1029/2009GL041814, 2010.

Ergun, R. E., L. Andersson, W. K. Peterson, D. Brain, G. T. Delory, D. L. Mitchell, R. P. Lin, and A. W. Yau, Role of plasma waves in Mars' atmospheric loss, Geophys. Res. Lett., 33, L14103, doi:10.1029/2006GL025785, 2006.

Fang, X., M. W. Liemohn, A. F. Nagy, Y. Ma, D. L. De Zeeuv, J. U. Kozyra, and T. H. Zurbuchen, Pickup oxygen ion velocity space and spatial distribution around Mars, J. Geophys. Res., 113, doi:10.1029/2007JA012736, 2008.

Fedorov, A., E. Budnik, J.-A. Sauvaud, C. Mazelle, S. Barabash, R. Lundin, M. Acũna, M. Holmström, A. Grigoriev, and M. Yamauchi, Structure of the Martian wake, Icarus, 182, 329-336, 2006.

Fränz, M., E. Dubinin, E. Roussos, J. Woch, J. D. Winningham, R. Frahm, A. J. Coates, A. Fedorov, S. Barabash, and R. Lundin, Plasma moments in the Environment of Mars. Mars Express ASPERA-3 observations, Space Sci. Rev., 126, 165-207, doi:10.1007/s11214-006-9115-9, 2006.

Fränz, M., E. Dubinin, E. Nielsen, J. Woch, S. Barabash, R. Lundin, and A. Fedorov, Transterminator ion flow in the Martian ionosphere, Planet. Space Sci., 58, 1442-1454, doi:10.1016/j.pss.2010.06.009, 2010.

Hoffman, N., White Mars: A new model for Mars' surface and atmosphere based on $\mathrm{CO}_{2}$, Icarus, 146, 326-342, doi:10.1006/icar.2000.6398, 2000.

Lammer, H., J. F. Kasting, E. Chassefière, R. E. Johnson, Y. N. Kulikov, and F. Tian, Atmospheric escape and evolution of terrestrial planets and satellites, Space Sci. Rev., 139, 399-436, 2008.

Luhmann, J. G., The solar wind interaction with unmagnetized planets-A tutorial, in Washington DC American Geophysical Union Geophysical Monograph Series, 58, 401-411, 1990.

Lundin, R., A. Zakharov, R. Pellinen, B. Hultqvist, H. Borg, E. Dubinin, S. Barabash, N. Pissarenko, H. Koskinen, and I. Liede, First results of the ionospheric plasma escape from Mars, Nature, 341, 609-612, 1989.

Lundin, R., S. Barabash, A. Fedorov, M. Holmström, H. Nilsson, J.-A. Sauvaud, and M. Yamauchi, Solar forcing and planetary ion escape from Mars, Geophys. Res. Lett., 35, L09203, doi:10.1029/2007GL032884, 2008a.

Lundin, R., S. Barabash, M. Holmström, H. Nilsson, M. Yamauchi, M. Fraenz, and E. M. Dubinin, A comet-like escape of ionospheric plasma from Mars, Geophys. Res. Lett., 35, doi:10.1029/ 2008GL034811, 2008b

Lundin, R., S. Barabash, M. Holmström, H. Nilsson, M. Yamauchi, E. M. Dubinin, and M. Fraenz, Atmospheric origin of cold ion escape from Mars, Geophys. Res. Lett., 36, L17202, doi:10.1029/2009GL039341, 2009.

Lundin, R., S. Barabash, M. Yamauchi, H. Nilsson, and D. Brain, On the relation between plasma escape and the Martian crustal magnetic field, Geophys. Res. Lett., 38, L02102, doi:10.1029/2010GL046019, 2011.

Mitchell, D., R. Lin, H. Rème, D. Crider, P. Cloutier, J. Connerney, M. Acuña, and N. Ness, Oxygen Auger electrons observed in Mars ionosphere, Geophys. Res. Lett., 27, 1871-1874, 2000.

Nilsson, H., E. Carlsson, H. Gunell, Y. Futaana, S. Barabash, R. Lundin, A. Fedorov, Y. Soobiah, A. Coates, M. Fränz, and E. Roussos, Investigation of the influence of magnetic anomalies on ion distributions at Mars, Space Sci. Rev., 126, 355-372, 2006a.

Nilsson, H., M. Waara, S. Arvelius, O. Marghitu, M. Bouhram, Y. Hobara, M. Yamauchi, R. Lundin, H. Rème, J.-A. Sauvaud, I. Dandouras, A. Balogh, L. M. Kistler, B. Klecker, C. W. Carlson, M. B. BavassanoCattaneo, and A. Korth, Characteristics of high altitude oxygen ion energization and outflow as observed by Cluster; a statistical study, Ann. Geophys., 24, 1099-1112, 2006b.

Nilsson, H., E. Carlsson, D. Brain, M. Yamauchi, M. Holmström, S. Barabash, R. Lundin, and Y. Futaana, Ion escape from Mars as a function of solar wind conditions: A statistical study, Icarus, 206, 4049, doi:10.1016/j.icarus.2009.03.006, 2010.

Nilsson, H., N. J. T. Edberg, G. Stenberg, S. Barabash, M. Holmström, Y. Futaana, R. Lundin, and A. Fedorov, Heavy ion escape from Mars, influence from solar wind conditions and crustal magnetic fields, Icarus, 215, 475-484, doi:10.1016/j.icarus.2011.08.003, 2011.

Slapak, R., H. Nilsson, M. Waara, M. André, G. Stenberg, and I. A. Barghouthi, $\mathrm{O}^{+}$heating associated with strong wave activity in the high altitude cusp and mantle, Ann. Geophys., 29, 931-944, doi:10.5194/angeo29-931-2011, 2011

Squyres, S. W., J. P. Grotzinger, R. E. Arvidson, J. F. Bell, W. Calvin, P. R. Christensen, B. C. Clark, J. A. Crisp, W. H. Farrand, K. E. Herkenhoff, 
J. R. Johnson, G. Klingelhöfer, A. H. Knoll, S. M. McLennan, H. Y. McSween, R. V. Morris, J. W. Rice, R. Rieder, and L. A. Soderblom, In situ evidence for an ancient aqueous environment at Meridiani Planum, Mars, Science, 306, 1709-1714, doi:10.1126/science.1104559, 2004.

Trotignon, J. G., C. Mazelle, C. Bertucci, and M. H. Acuna, Martian shock and magnetic pile-up boundary positions and shapes determined from the Phobos 2 and Mars Global Surveyor data sets, Planet. Space Sci., 54, 357-369, 2006.

Verigin, M. I., N. M. Shutte, A. A. Galeev, K. I. Gringauz, G. A. Kotova, A. P. Remizov, H. Rosenbauer, P. Hemmerich, S. Livi, A. K. Richter, I. Apathy, K. Szego, W. Riedler, K. Schwingenschuh, M. Steller, and Y. G. Yeroshenko, Ions of planetary origin in the Martian magnetosphere (Phobos 2/TAUS experiment), Planet. Space Sci., 39, 131-137, 1991.

Vignes, D., C. Mazelle, H. Rème, M. Acuña, J. Connerney, R. Lin, D. Mitchell, P. Cloutier, D. Crider, and N. Ness, The solar wind interaction with Mars: locations and shapes of the bow shock and magnetic pile-up boundary from the observations of the MAG/ER experiment onboard Mars Global Surveyor, Geophys. Res. Lett., 27, 49-52, 2000.

Waara, M., S. Slapak, H. Nilsson, G. Stenberg, M. André, and I. A. Barghouthi, Statistical evidence for $\mathrm{O}^{+}$energization and outflow caused by wave-particle interaction in the high altitude cusp and mantle, Ann. Geophys., 29, 945-954, doi:10.5194/angeo-29-945-2011, 2011.

Yamauchi, M., Y. Futaana, A. Fedorov, R. A. Frahm, J. D. Winningham, E. Dubinin, R. Lundin, S. Barabash, M. Holmström, C. Mazelle, J. Sauvaud, T. L. Zhang, W. Baumjohann, A. J. Coates, and M. Fraenz, Comparison of accelerated ion populations observed upstream of the bow shocks at Venus and Mars, Ann. Geophys., 29, 511-528, doi:10.5194/angeo-29-511-2011, 2011.

H. Nilsson (e-mail: hans.nilsson@irf.se), G. Stenberg, Y. Futaana, M. Holmström, S. Barabash, R. Lundin, N. J. T. Edberg, and A. Fedorov 